\title{
Decreased circulating ErbB4 ectodomain fragments as a read-out of impaired signaling function in amyotrophic lateral sclerosis
}

\section{Abbreviated title: Decreased ecto-ErbB4 in ALS}

Lopez-Font I, Sogorb-Esteve A, Javier-Torrent M, Brinkmalm G, Herrando-Grabulosa M, García-Lareu B, Turon-Sans J, Rojas-García R, Lleó A, Saura CA, Zetterberg H, Blennow

$$
\text { K, Bosch A, Navarro X, Sáez-Valero J. }
$$

${ }^{1}$ Instituto de Neurociencias de Alicante, Universidad Miguel Hernández-CSIC, Sant Joan d'Alacant, Spain; ${ }^{2}$ Centro de Investigación Biomédica en Red sobre Enfermedades Neurodegenerativas (CIBERNED), Spain; ${ }^{3}$ Institute of Neurosciences and Department of Biochemistry and Molecular Biology, Universitat Autònoma de Barcelona, Bellaterra, Spain; ${ }^{4}$ Clinical Neurochemistry Laboratory, Sahlgrenska University Hospital, Mölndal, Sweden; ${ }^{5}$ Institute of Neuroscience and Physiology, University of Gothenburg, Mölndal Campus, Sweden; ${ }^{6}$ Institute of Neurosciences and Department of Cell Biology, Physiology and Immunology, Universitat Autònoma de Barcelona, Bellaterra, Spain; ${ }^{7}$ Neuromuscular Diseases Unit, Department of Neurology, Hospital de la Santa Creu i Sant Pau, Universitat Autònoma de Barcelona, Barcelona, Spain; ${ }^{8}$ Centro de Investigación Biomédica en Red de Enfermedades Raras (CIBERER), Spain; ${ }^{9}$ Memory Unit, Neurology Department, Hospital de la Santa Creu i Sant Pau, Barcelona, Spain; ${ }^{10}$ Department of Neurodegenerative Disease, Institute of Neurology, University College London, London, UK; ${ }^{11}$ UK Dementia Research Institute at UCL, London, UK.

$\dagger$ A. Sogorb-Esteve and I. Lopez-Font contributed equally to this study. 
*To whom correspondence should be addressed:

Javier Sáez-Valero, Instituto de Neurociencias de Alicante, Universidad Miguel

Hernández-CSIC, Av. Ramón y Cajal s/n, 03550 Sant Joan d'Alacant, Spain.

Phone: +34 965919580; Fax: +34 965919561; email: j.saez@umh.es

Number of Figures/Tables: 6 Figures +1 Supplemental Figure +1 Supplemental Table

Abstract: 280 words / Full-length: 5000 words 


\section{Abstract}

ErbB4 is a transmembrane receptor tyrosine kinase that binds to neuregulins to activate signaling. Proteolytic cleavage of ErbB4 results in release of soluble fragments of ErbB4 into the interstitial fluid. Disruption of the neuregulin-ErbB4 pathway has been suggested to be involved in the pathogenesis of amyotrophic lateral sclerosis (ALS). This study assesses whether soluble proteolytic fragments of the ErbB4 ectodomain (ecto-ErbB4) can be detected in cerebrospinal fluid (CSF) and plasma, and if the levels are altered in ALS. Immunoprecipitation combined with mass spectrometry or western blotting analyses confirmed the presence of ecto-ErbB4 in human CSF. Several anti-ErbB4-reactive bands, including a $55 \mathrm{kDa}$ fragment, were detected in CSF. The bands were generated in the presence of neuregulin-1 (Nrg1) and were absent in plasma from ErbB4 knockout mice. Ecto-ErbB4 levels were decreased in CSF from ALS patients $(n=20)$ and ALS with concomitant frontotemporal dementia patients $(n=10)$, compared to age-matched controls $(n=13)$. A similar decrease was found for the short ecto-ErbB4 fragments in plasma of the same subjects. Likewise, the 55-kDa ecto-ErbB4 fragments were decreased in the plasma of the two transgenic mouse models of ALS (SOD1G93A and TDP-43A315T). Intracellular ErbB4 fragments were decreased in the frontal cortex from SOD1G93A mice, indicating a reduction in Nrg-dependent induction of ErbB4 proteolytic processing, and suggesting impaired signaling. Accordingly, overexpression of $\mathrm{Nrg} 1$ induced by an adeno-associated viral vector increased the levels of the ecto-ErbB4 fragment in the SOD1G93A mice. We conclude that the determination of circulating ecto-ErbB4 fragments could be a tool to evaluate the impairment of the ErbB4 pathway and may be a useful biomarker in ALS. 
Key Words: Amyotrophic lateral sclerosis; ErbB4; biomarker; cerebrospinal fluid; brain; plasma; transgenic model. 


\section{Introduction}

ErbB4 (Erb-B2 Receptor Tyrosine Kinase 4) is a single-pass type I transmembrane receptor that contains a cytoplasmic tyrosine kinase domain (Plowman et al., 1993). This member of the epidermal growth factor (EGF) receptor subfamily binds and is activated by neuregulins (Nrg1, Nrg2, Nrg3 and Nrg4), heparin-binding EGF-like growth factor and other members of the EGF family such as betacellulin, inducing a variety of cellular responses including cell proliferation and differentiation (reviewed in Carpenter, 2003; Roskoski, 2014). Ligandbinding triggers receptor dimerization and autophosphorylation at specific tyrosine residues that then serve as binding sites for scaffold proteins and effectors transducing cellular responses.

Disruption of the neuregulin-ErbB4 pathway has been involved in the pathogenesis of amyotrophic lateral sclerosis (ALS), in which loss-of-function mutations on ERBB4 produce late-onset ALS in human patients (Takahashi et al., 2013). ALS is a complex multisystem neurodegenerative disease seen in comorbidity with a wide range of cognitive and behavioral abnormalities, ranging 15 to $50 \%$ of cases (Montuschi et al., 2015; Cui et al., 2015), and particularly overlapping with frontotemporal dementia (FTD) (Lillo and Hodges, 2010). Possible pathogenic mutations in ERBB4 gene has been also recently described in concomitant ALS and FTD (Dols-Icardo et al., 2017).

Nrg1-binding induces activation of ErbB4, but also the ensuing sequential proteolytic cleavage of the receptor (Sardi et al., 2006), firstly by the metalloprotease ADAM17 (TNF-a-converting enzyme; TACE), which causes the secretion of an N-terminal ecto-domain fragment (ecto-ErbB4) (Rio et al. 2000). The remaining C-terminal fragment (ErbB4-CTF) is processed by $\gamma$-secretase/presenilin1 (PS1), generating shorter intracellular 
domain (ErbB4-ICD) fragments (Ni et al., 2001). This ErbB4-ICD fragment can translocate to the nucleus retaining tyrosine kinase activity (Vecchi and Carpenter 1997; Bao et al., 2013), and regulates gene expression (Sardi et al., 2006; Ancot et al., 2009). Other metalloproteases, identified in various cell types, can also promote ErbB receptor ectodomain cleavage (Yu et al., 2002; Lynch et al., 2007; Sanderson et al. 2006). The soluble extracellular ecto-ErbB4 fragments, resulting from metalloprotease cleavage, also exhibit biological activity, binding Nrg1 and blocking autocrine and paracrine signaling (Woo et al., 2007; Ma et al., 2009; Wen et al., 2010; Tian et al., 2017). Thus, ecto-ErbB4 has been suggested to influence brain activity, regulating synaptic plasticity (Chen et al., 2010), anxiety-like behavior (Geng et al., 2016), and furthermore exhibiting potential as a drug candidate (Gambarotta et al., 2015; Pascal et al., 2014). These soluble ecto-ErbB4 fragments are expected to be present in circulatory fluids (Baron et al., 2009). Indeed, ecto-ErbB4 fragments have been detected in the medium of cultured cancer cells (Hollmén et al., 2009; 2012). However, to our knowledge, the levels of circulating ecto-ErbB4 have not yet been assessed in CSF and/or plasma of patients suffering neurodegenerative disorders.

In this study, we investigated if truncated soluble ecto-ErbB4 is detectable in CSF and plasma of ALS and ALS-FTD patients and in plasma of a transgenic ALS mouse model. We have characterized a short 55-kDa ecto-ErbB4 fragment in plasma and CSF and found reduced levels in ALS. Additionally, this 55-kDa ecto-ErbB4 fragment increased in parallel to increasing Nrg1 type III expression. Moreover, in the brain of ALS transgenic mice, we also addressed an impairment in the ErbB4 proteolytic processing rate, indicative of failure in this signaling pathway. 


\section{Materials and methods}

\section{Patients, SOD1 ${ }^{\text {G93A }}$ transgenic mice, ErbB4 knock-out mice and ErbB4/Nrg1 over- expressing cells}

This study was approved by the local ethics committees of all the centers involved and it was carried out in accordance with the Helsinki Declaration.

Study participants: Patients with ALS, and availability of CSF and plasma samples, were selected from the Motor Neuron Disease Clinic at the Hospital de la Santa Creu i Sant Pau, Barcelona, Spain. Patients included in the study fulfilled El Escorial revised criteria for probable, probable laboratory-supported or definite ALS (Brooks et al., 2000). All of them underwent a cognitive and behavioural screening and classified as ALS without cognitive or behavioral impairment and ALS-FTD accordingly to reported criteria (Strong et al., 2017). Participants included 20 ALS patients (11 men and 9 women, $67 \pm 5$ years), 15 of them without cognitive and behavioral abnormalities ( 8 men and 7 women, $65 \pm 6$ years) and 5 ALS with concomitant FTD (ALS-FTD; 3 men and 2 women, $72 \pm 8$ years) (Illan-Gala et al., 2018). Age-matched cognitively normal controls were randomly selected from the Sant Pau Initiative on Neurodegeneration (SPIN cohort: https://santpaumemoryunit.com/ourresearch/spin-cohort/) (Alcolea et al., 2017). Controls had no history or symptoms of neurological or psychiatric disorders, or memory complaints (6 men and 4 women, $68 \pm 1$ years) and had normal levels of core AD biomarkers in CSF (Alcolea et al., 2015). Plasma samples were from the same individuals, except an ALS case $(n=19 ; 10$ men and 9 women, $67 \pm 5$ years). Samples were collected in heparinized tubes and separated from whole blood by centrifugation at $3000 \times \mathrm{g}$ for $15 \mathrm{~min}$ at $4^{\circ} \mathrm{C}$. CSF and plasma samples were aliquoted and frozen at $-80^{\circ} \mathrm{C}$ until use. 
Material collected from animals: Animal care and experimental procedures were approved by the Biosafety and the Ethical Committees of the Universitat Autònoma de Barcelona. Plasma samples were collected from ten 12 weeks-old of the mutant (G93A) superoxide dismutase 1 (SOD1) mouse model (B6SJL-Tg[SOD1-G93A]1Gur; obtained from the Jackson Laboratory), the most widely used transgenic mouse ALS model, and ten wild-type littermate. The SOD1 ${ }^{\mathrm{G} 93 \mathrm{~A}}$ transgenic mice recapitulates the most relevant clinical and histopathological features of both familial and sporadic ALS, developing rapidly progressive motoneuron loss, with locomotor deficits from 12 to 13 weeks of age, hindlimb weakness and muscle atrophy, culminating in paralysis and death between ages 16 and 19 weeks (Ripps et al., 1995; Mancuso et al., 2011). Upon anesthesia by isofluorane, blood was withdrawn after decapitation in an EDTA tube and centrifuged at $1000 \times \mathrm{g}$ for 10 min at $4^{\circ} \mathrm{C}$. The supernatant was aliquoted and stored at $-80^{\circ} \mathrm{C}$ until further analysis.

For analysis of the proteolytic processing of ErbB4 in the SOD1 $1^{\mathrm{G} 93 \mathrm{~A}}$ mice, the brains from six 12 weeks-old transgenic mice and five littermates were removed and the cerebral hemispheres were dissected out discarding brain stem and cerebellum. Both brain hemispheres were homogenized with a Dounce-type glass homogenizer in ice-cold buffer containing $10 \mathrm{mM}$ Hepes pH7.5, $1.5 \mathrm{mM} \mathrm{MgCl} 2,10 \mathrm{mM} \mathrm{KCl}, 1 \mathrm{mM} \mathrm{NaVO} 4,25 \mathrm{mM} \mathrm{NaF}$, $200 \mathrm{mM}$ sucrose, 10\% NP-40 (w/v), $0.5 \mathrm{mM}$ PMSF (phenylmethylsulfonyl fluoride) and supplemented with the protease inhibitor 5mM PNT (1,10-Phenanthroline monohydrate from Sigma-Aldrich Co). Then, the homogenates were sonicated and frozen at $-80^{\circ} \mathrm{C}$ until use. Total protein concentrations were determined using the bicinchoninic acid method (BCA from Pierce). 
$A A V$ vector construction, production and titration: The cDNA sequence of the extracellular domain of Nrg1 type III isoform (Nrg1-III) was kindly provided by G. Corfas (Harvard Medical School, Boston, MA) and cloned between AAV2 ITRs under the regulation of the CMV promoter. The woodchuck hepatitis virus responsive element (WPRE) was added at 3' to stabilize mRNA expression (Loeb et al., 1999). AAVrh10 viral stocks were produced by the Viral Production Unit of Universitat Autònoma de Barcelona (http://viralvector.eu) as previously described (Zolotukhin et al., 1999) by triple transfection into HEK293-AAV cells of the expression plasmid, Rep2Caprh10, containing AAV genes (kindly provided by J.M. Wilson, University of Pennsylvania, Philadelphia, USA) and pXX6 plasmid containing adenoviral genes (Xiao et al., 1998), needed as helper virus. AAV particles were purified by iodixanol gradient. Titration was evaluated by picogreen (Invitrogen) quantification (Piedra et al., 2015) and calculated as viral genomes per milliliter (vg/ml). Control serotype-matching AAVrh10 empty vector was used as control (mock).

Animal surgery: Intrathecal administration was performed as described (Homs et al., 2014). Briefly, eight 8 weeks-old $\mathrm{SOD} 1^{\mathrm{G} 93 \mathrm{~A}}$ mice were anesthetized by intraperitoneal administration of ketamine $(10 \mathrm{mg} / \mathrm{kg}$ of body weight; Imalgene $50 \mathrm{mg} / \mathrm{mL}$; Merial Laboratorios) and xylacine (1 mg/kg of body weight; Rompun; Bayer AG). After exposing the lumbar L3 and L4 vertebrae, $7.6 \times 10^{10} \mathrm{vg}$ of an adeno-associated viral vector (AAV) encoding for soluble the ectodomain extracellular domain (ECD) of Nrg1, in a total volume of $10 \mu \mathrm{l}$, were slowly delivered into the intervertebral foramen using a Hamilton syringe and a 33 Gauge needle (Hamilton Company). Proper position of the needle was confirmed by a tail flick reflex. The needle was removed 30 seconds after the end of vector delivery to favor spread through the CSF and avoid efflux to periphery. After injection, epaxial muscles were 
sutured and the skin closed with suture clips. All mice were fed ad libitum with a standard diet (Teklad Global, Harlan Teklad). Plasma samples were obtained, at sacrifice 8 weeks after the intrathecal AAV-Nrg1.III administration.

Plasma samples were also collect from P50 ErbB4 knockout mice (ErbB4-KO kindly provided by Dr. B. Rico from Centre for Developmental Neurobiology, Institute of Psychiatry, Psychology and Neuroscience, King's College London, London, UK; see Tidcombe et al. 2003) and littermates for probing the specificity of the immunoreactive bands.

Material collected from cells: HEK293 cells $\left(1.5 \times 10^{6}\right.$ cells/well) were grown in $60 \mathrm{~mm}$ plates in Dulbecco's modified Eagle's medium (DMEM; Sigma-Aldrich Co) supplemented with 5\% fetal bovine serum (FBS; Gibco) and $100 \mu \mathrm{g} / \mathrm{mL}$ penicillin/streptomycin (Gibco). After $24 \mathrm{~h}$, the medium was recollected and cells washed twice with phosphate buffered saline (PBS), to eliminate rest of albumin, and were transferred to a modified Eagle's Minimum Essential Media, OptiMEM (Gibco; supplemented with glutamine (2 mM), which allow maintenance of cells without serum. Cells in OptiMEM were transfected with a construct encoding full-length ErbB4 JM-a splicing variant (Gambarotta et al., 2004), and alternatively co-transfected with a plasmid encoding Nrg1-III [EF1-Nrg1a Type III Flag (Mancuso et al, 2016)], using Lipofectamine 2000 (Thermo Fisher Scientific), according to the manufacturer's instructions. Cell media was changed, and after $48 \mathrm{~h}$ of transfection, cells were mechanically lysed in cold lysis buffer [50 mM Tris- $\mathrm{HCl}$ pH 7.4, $150 \mathrm{mM} \mathrm{NaCl,} 2 \mathrm{mM}$ EDTA, 1\% NP-40, $1 \mathrm{mM}$ PMSF supplemented with phosphatase and protease inhibitors (Roche)], incubated for $30 \mathrm{~min}$ on ice, centrifuged for $10 \mathrm{~min}\left(10,000 \mathrm{rpm}, 4^{\circ} \mathrm{C}\right)$ and the supernatant was kept. Conditioned media was supplemented with PMSF inhibitor (1 mM), 
centrifuged at 1,000 rpm for 5 min at RT, and concentrated using Amicon® Ultra 10K filters (EMD Millipore), according to the manufacturer's instructions. Both, lysates and conditioned media was resolved on $10 \%$ SDS-PAGE gel and analyzed by for N-terminal and C-terminal ErbB4 fragments by western blotting.

Western blotting, immunoprecipitation and preparation of the samples for enzymatic deglycosylation and mass spectrometric data analysis

For western blotting, CSF $(30 \mu \mathrm{L})$ and plasma samples $(0.4 \mu \mathrm{L})$, mouse brain extracts (40 $\mu \mathrm{g})$, HEK293 extracts $(25 \mu \mathrm{g})$ and supernatants $(25 \mu \mathrm{L})$ were denatured at $65^{\circ} \mathrm{C}$ for 5 min and resolved by electrophoresis on $10 \%$ SDS-polyacrylamide gels. Following electrophoresis, proteins were blotted onto nitrocellulose membranes (Bio-Rad Laboratories, Inc.) and ErbB4 was detected with antibodies against the $\mathrm{N}$-terminal part as indicated, a polyclonal antibody (from Thermo Fisher Scientific Inc.), the immunogen of which is a synthetic peptide between 25-35 amino acids from the N-terminal region of human ErbB4, a monoclonal ErbB4 antibody (clone Ab77; Thermo Fisher Scientific Inc.), the immunogen of which is an extracellular fragment of recombinant human ErbB4, or an antibody against the C-terminal part of the protein (polyclonal antibody C-18 from Santa Cruz). GAPDH (Ambion, Life Technologies) was used as a loading control for brain extracts. Antibody binding was visualized with fluorescent secondary IRDye antibodies and recorded on an Odyssey CLx Infrared Imaging system (LI-COR Biosciences GmbH).

Immunoprecipitations were performed at $4^{\circ} \mathrm{C}$ by incubating $200 \mu \mathrm{L}$ of $\mathrm{CSF}$, or $2 \mu \mathrm{L}$ of plasma, overnight with the polyclonal ErbB4 antibody, or alternatively as a control with an irrelevant rabbit IgG, previously coupled to protein A-Sepharose by Dimethyl 
pimelimidate dihydrochloride (Sigma-Aldrich Co). Precipitated proteins were washed with PBS and eluted with $0.1 \mathrm{M}$ glycine buffer at $\mathrm{pH} 2.5$. After $\mathrm{pH}$ neutralization, supernatants were denatured in Laemmli sample buffer at $50^{\circ} \mathrm{C}$ for $15 \mathrm{~min}$ and subjected to SDS-PAGE. Separated proteins were transferred to nitrocellulose membranes and blotted with the monoclonal N-terminal antibody.

Immunoprecipitation was also performed for further analysis by high resolution mass spectrometry. For this purpose, immunoprecipitation (IP) of $5 \mathrm{~mL}$ of CSF was performed using a KingFisher magnetic particle processor (Thermo Fisher Scientific Inc.), according to a previously described protocol (Portelius et al., 2010) with minor modifications. The polyclonal ErbB4 antibody was again used to immunoprecipitate ErbB4. Briefly, $4 \mu \mathrm{g}$ of the antibody was cross-linked to $50 \mu \mathrm{L}$ magnetic Dynabeads M-280 Sheep anti-mouse IgG (Invitrogen). Beads with antibodies were incubated in CSF overnight. Eluates $(100 \mu \mathrm{L}$ in $0.5 \%$ formic acid) were vacuum-dried and stored at $-80{ }^{\circ} \mathrm{C}$ pending further handling.

In-solution enzymatic digestion: Immunoprecipitated samples were digested in-solution with trypsin (Sequencing grade modified trypsin, Promega, Madison, WI, USA) as follows: The dried eluates were dissolved in $25 \mu \mathrm{L} 50 \mathrm{mM}$ ammonium bicarbonate (Riedel-de Haën, Seelze, Germany) and allowed to shake for $1 \mathrm{~h}$. Reduction was made by adding $5 \mu \mathrm{L} 20 \mathrm{mM}$ dithiothreitol (Sigma-Aldrich) in $50 \mathrm{mM}$ ammonium bicarbonate followed by incubation 30 min at $60{ }^{\circ} \mathrm{C}$. The solutions were cooled to room temperature and alkylation was performed by adding $5 \mu \mathrm{L} 75 \mathrm{mM}$ iodacetamide (Sigma-Aldrich) in $50 \mathrm{mM}$ ammonium bicarbonate followed by incubation $30 \mathrm{~min}$ in darkness. One $\mu \mathrm{g}$ trypsin was dissolved in $400 \mu \mathrm{L} 50 \mathrm{mM}$ ammonium bicarbonate ( $\mathrm{pH}$ 8.5). Five $\mu \mathrm{L}$ trypsin solution was added followed by incubation over night at $37{ }^{\circ} \mathrm{C}$. To stop the enzymatic activity $2 \mu \mathrm{L} 10 \%$ aqueous trifluoroacetic acid (Sigma-Aldrich) was added followed by incubation $45 \mathrm{~min}$ at $37^{\circ} \mathrm{C}$, after which the solution 
was centrifuged $10 \mathrm{~min}$ at $16910 \times \mathrm{g}$. Samples were then vacuum-dried and stored at $-80{ }^{\circ} \mathrm{C}$ pending mass spectrometric analysis.

LC-MS/MS: Mass spectrometric analysis was performed using a Dionex 3000 nanoflow LC system coupled to a Q Exactive (both Thermo Fisher Scientific), a hybrid electrospray ionization linear quadrupole-Orbitrap mass spectrometer. Briefly, a reversed phase Acclaim

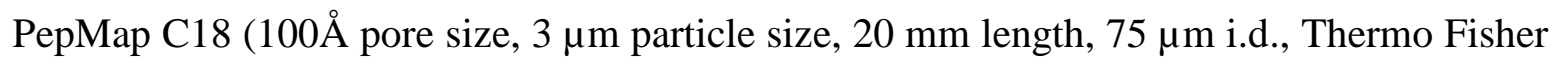
Scientific) trap column was used for online desalting and sample clean-up, followed by a reversed phase Acclaim PepMap RSLC C18 (100 Å pore size, $2 \mu \mathrm{m}$ particle size, $75 \mu \mathrm{m}$ i.d., $150 \mathrm{~mm}$ length, Thermo Fisher Scientific) for separation. The separation was performed at a flow rate of $300 \mathrm{~nL} / \mathrm{min}$. by applying a linear gradient of $0-40 \% \mathrm{~B}$ for $50 \mathrm{~min}$. Mobile phase A was $0.1 \%$ formic acid in water (v/v) and mobile phase B was $0.1 \%$ formic acid and $84 \%$ acetonitrile in water (v/v/v). All mass spectra were acquired data dependent in positive ion mode with a resolution setting of 70000 for precursor ion acquisitions and 17500 fragment mass spectra. Precursor fragmentation was obtained by higher energy collision induced dissociation (HCD) using a normalised collision energy (NCE) setting of 28.

Mass spectrometric data analysis: Database searches were performed for screening purposes using Proteome Discoverer (v2.1, Thermo Fisher Scientific) and submitted to an in-house Mascot database server (v.2.6, Matrix Science, London, UK). Spectra were searched against a Uniprot human database using $10 \mathrm{ppm}$ mass accuracy for precursor ions and $50 \mathrm{mDa}$ for fragment ions.

Enzymatic deglycosylation: Serum glycoproteins were treated with an enzymatic deglycosylation kit from ProZyme (GK80110) following manufacturer indications, and then 
subjected to SDS-PAGE and western blot analysis. This treatment remove all N-linked glycans and simple O-linked glycans (including polysialylated) from glycoproteins.

\section{Statistical analysis.}

All data were analyzed by one-way analysis of variance (ANOVA), or through a Student's $t$ test (two-tailed) for single pair-wise comparisons, determining the exact $p$ values. When normality was rejected, a Mann-Whitney Rank Sum Test was used. The results are presented as the means \pm SEM and all the analyses were all performed using SigmaStat (Version 2.0; SPSS Inc.). Correlations between variables were assessed by linear regression analyses. A $p$ value $<0.05$ was considered significant. 


\section{Results}

\section{Ecto-ErbB4 fragments are present in CSF and plasma}

ErbB4 is a 1308 amino acid-long type I glycoprotein which extracellular and intracellular domains compromise similar number of amino acids (Fig. 1A). To determine the presence of ecto-ErbB4 fragment in human CSF, we first examined the samples by immunoprecipitation/western blot analysis using a polyclonal anti N-terminal antibody. Several immunoreactive bands for the N-terminus of ErbB4, between 55 and $80 \mathrm{kDa}$, were detected in human CSF (Fig. 1B). CSF samples were also immunoprecipitated using the polyclonal anti-ErbB4 antibody. Mass spectrometry analysis of the immunoprecipitates identified eighteen tryptic peptides spanning aa 36-606 of human ErbB4 (Uniprot entry Q15303, ERBB4_HUMAN) in CSF, matching sequences located from the N-terminus of the protein until the transmembrane domain (Fig 1A and supplemental Table 1). Western blot analysis of the immunoprecipitates with an alternative monoclonal antibody which immunogen is an extracellular fragment of recombinant human ErbB4 (clone Ab77) confirmed the nature of $55 \mathrm{kDa}$ ecto-ErbB4 fragments, with a faint $80 \mathrm{kDa}$ band (Fig 1B). An $80 \mathrm{kDa}$ band was also resolved in the unbound fraction enriched in a non-specific 70 kDa band which display also positive immunoreactivity to an anti-albumin antibody (not shown). The 55 and $80 \mathrm{kDa}$ bands were not observed in negative immunoprecipitation controls with an irrelevant antibody (Fig 1B).

These ecto-ErbB4 fragments were also detected in human plasma with the shortest resolved as a double set of bands between 55 and $50 \mathrm{kDa}$ (Fig 1C). Both shortest ectoErbB4 fragment were also resolved in immunoprecipitates, while the $80 \mathrm{kDa}$ band was not resolved (Fig 1C). To assess whether the doublet of ecto-ErbB4 fragments represent similar 
fragments which difference in molecular mass derived from differential glycosylation, we determined the effect of enzymatic deglycosylation on the electrophoretic migration of ecto-ErbB4 fragments in human plasma. After treatment with glycosidases, both the 50 and $55 \mathrm{kDa}$ bands suffered a reduction in apparent molecular weight but remained as a doublet band, indicating that original differences in molecular weight are not due to differences in glycosylation (Fig 1D).

In mouse plasma, the doublet of shortest ecto-ErbB4 fragments was also apparent, but poorly resolved in most samples (Fig 2A). The shorter doublet of ecto-ErbB4 fragments was the only immunoreactive band absent in plasma from an ErbB4-KO mouse model (Tidcombe et al., 2003), which display weak immunoreactivity for the $80 \mathrm{kDa}$ band (Fig 2A). Moreover, to confirm the specificity of the short 55 ecto-ErbB4 fragment and if this fragment is generated subsequently to the binding of neuregulins, we first over-expressed ErbB4 in the HEK293 cell line and then examined whether Nrg1 induces the generation of these $55 \mathrm{kDa}$ ecto-ErbB4 fragment in the cellular system. HEK293 cells, maintained in OptiMEM medium (supplemented with glutamine, but not with FBS, to avoid the interference of albumin) were transfected with a construct encoding the full-length cleavable ErbB4 JM-a splicing variant (Gambarotta et al., 2004), susceptible to metalloprotease/secretase proteolytic processing (Määttä et al., 2006). The over-expression of ErbB4 in HEK293 cells served to probe the generation of both larger and short ectoErbB4 fragments, in parallel to C-terminal fragments in cell extracts (Fig 2B). When HEK293 cells were co-transfected with a construct encoding Nrg1-III an increase generation of shorter ecto-ErbB4 fragments was observed in the cell medium (Fig 2B).

Altogether, we confirmed the presence of circulating shortest ecto-ErbB4 fragments of $\sim 55 \mathrm{kDa}$ bands that can be generate after binding with Nrg1. Some of the 
immunoreactivity of the larger $80 \mathrm{kDa}$ band overlap with unspecific reactivity, at least in plasma, which can compromise a reliable quantification.

\section{Ecto-ErbB4 fragments are decreased in ALS and ALS-FTD CSF and plasma}

To assess whether levels of ecto-ErbB4 fragments are altered in ALS and ALS-FTD subjects, we first analyzed CSF samples from 20 ALS patients, including 5 ALS-FTD, and in 10 age-matched control samples (Ctrl). The 80 and 55 ecto-ErbB4 fragments were detected with the polyclonal N-terminal antibody in all CSF analyzed (Fig 3A). Regarding the previous analyses of specificity, we decided to consider the immunoreactivity levels for the short 55 band, which decreased $(38 \% ; p=0.037)$ in the pathological samples compared to Ctrl subjects (Fig 3B). In the sub-group of pathological samples, the decrease was particularly relevant for the ALS-FTD subjects (76\% decrease; $p=0.032$ ) than for ALS subjects which alone failed to be statistically significant $(26 \%$ decrease; $p=0.14)$. The 80 kDa large ecto-ErbB4 fragment also decreased significantly, in similar extension for the ALS without concomitant FTD (27\% decrease; $p=0.012)$ and for the ALS-FTD subjects (32\% decrease; $p=0.046$ ), compared to Ctrls (Fig 3B). Immunoreactivity levels of the 80 and $55 \mathrm{kDa}$ ecto-ErbB4 fragment correlated for Ctrl samples $(\mathrm{R}=0.86 ; p=0.002$; Supplemental Fig. 1A), but the correlation failed when pathological samples were considered (Supplemental Fig. 1A).

Plasma samples from 19 of the ALS, including the 5 ALS-FTD, and the 10 agematched Ctrl, were also available and ecto-ErbB4 fragments were determined. In human plasma was possible to discriminate and quantify separately the doublet band of 50 and 55 $\mathrm{kDa}$ for the shortest ecto-ErbB4 (Fig 4A). The 50 and $55 \mathrm{kDa}$ band did not correlate in Ctrl nor in ALS samples (Supplemental Fig. 1B). The immunoreactivity for the band displaying 
$\sim 50 \mathrm{kDa}$ was not different in ALS from Ctrl subjects; while, in good agreement with changes in CSF, for the $55 \mathrm{kDa}$ there was a significant decrease in the pathological group (43\%; $p=0.041$ ), with respect to that from Ctrl subjects (Fig 4B). Again, when considered separately the sub-group of pathological samples, this decrease was only significant in $\operatorname{ALS}-F T D(54 \% ; p=0.032)$, but not in ALS subjects (40\%; $p=0.12)$ (Fig 4B). The largest ecto-ErbB4 fragments were also resolved as a $\sim 80$ and $85 \mathrm{kDa}$ doublet, which did not display differences in any of the groups (Fig 4B). The 80-85 kDa doublet correlated only in Ctrl samples ( $\mathrm{R}=0.71 ; p=0.02$; Supplemental Fig. 1B), but not in the ALS (Supplemental Fig. 1B). No significant correlations were observed between largest and shortest ectoErbB4 fragment in plasma from Ctrl nor ALS subjects (not shown). Note that, at least in mice plasma, the $\sim 80 \mathrm{kDa}$ band overlap with unspecific immunoreactivities (Fig 1E); thus it is not possible to discern if these largest ecto-ErbB4 fragment are or are not affected, and for further analysis in the ALS transgenic mouse model we consider only the estimations of shortest ecto-ErbB4 fragments.

A positive correlation has found between the 55-kDa bands from CSF samples and the 55-kDa band from plasma in the pathological samples, indicating a similar shift in shortest ecto-ErbB4 levels in both fluids ( $\mathrm{R}=0.46 ; p=0.048$; Supplemental Fig $1 \mathrm{C})$, while the ecto-ErbB4 fragments were not correlated in Ctrl samples (Supplemental Fig 1C).

\section{Impaired generation of ecto-ErbB4 fragments in SOD1 ${ }^{\mathrm{G} 93 \mathrm{~A}}$ transgenic mice}

To determine whether a pathogenic SOD1 mutation may influence the levels of ecto-ErbB4 we examined by western blot the plasma of 12 weeks-old SOD $1^{\mathrm{G} 93 \mathrm{~A}}$ transgenic mice (Fig

5A). Immunoblot with the N-terminal ErbB4 antibody revealed that plasma from SOD1 ${ }^{\text {G93A }}$ mice had lower levels of the shortest ecto-ErbB4 doublet compared to those of control 
littermates (52\% decrease; $p=0.006$; Fig 5B). In mouse plasma, it was not possible to discriminate and quantify the doublet band separately.

We have demonstrated that $\mathrm{Nrg} 1$ binding induces in vitro the subsequent cleavage of ErbB4, generating shortest ecto-ErbB4 fragments. We further addressed in vivo the association of shortest ecto-ErbB4 fragments with $\mathrm{Nrg} 1$ using intrathecal injection of viral genomes of an AAV encoding for the extracellular domain (ECD) of Nrg1 in the SOD1 ${ }^{\mathrm{G} 93 \mathrm{~A}}$ transgenic mice. We verified a positive correlation in the plasma of injected Nrg1-ECD $\mathrm{SOD} 1^{\mathrm{G} 93 \mathrm{~A}}$ mice between the levels of soluble $\mathrm{Nrg} 1$ and shortest ecto-ErbB4 fragments $(\mathrm{R}=$ $0.80, p=0.018 ;$ Fig 5C, D).

Finally, we attempted to discard whether decreased plasma ecto-ErbB4 fragments in the SOD $1^{\mathrm{G} 93 \mathrm{~A}}$ transgenic mice, which can represent a read-out of impaired Nrg1/ErbB4 signaling function, is only a direct consequence of decreased ErbB4 expression levels. Indeed, significant decreased ErbB4 mRNA and protein levels in muscles of ALS patients have been reported (Mancuso et al., 2016), and similar reduction in ErbB4 levels are described in muscles (Mancuso et al., 2016), and the ECD of ErbB4 in motor neurons of the transgenic SOD1 ${ }^{\mathrm{G} 93 \mathrm{~A}}$ (Lasiene et al., 2016). Thus, we decided to determine the levels of unprocessed full-length of ErbB4 (ErbB4-fl) and intracellular fragments in brain as an indicator of affectation on the processing rate of the receptor and potential impairment in ErbB4 signaling. The ErbB4-fl and their intracellular C-terminal fragments (CTF) were resolved and quantified by western blot in brain extracts from transgenic SOD $1^{\mathrm{G} 93 \mathrm{~A}}$ and control littermates (Fig 6). A decreased ErbB4-CTF/ErbB4-fl quotient in SOD1 ${ }^{\text {G93A, }}$, without significant changes in ErbB4-fl, indicates a decreased proteolytic processing of ErbB4. Accordingly, we propose that decrease in circulating ecto-ErbB4 is likely due, at least in part, to impair signaling and subsequent proteolytic processing of the receptor. 


\section{Discussion}

ErbB4 full-length glycoprotein has a size of 1308 amino acids exhibiting a molecular mass of $\sim 160-180 \mathrm{kDa}$ depending of its glycosylation. Ligand interaction with ErbB4 initiates phosphorylation-dependent signaling cascades, but also, as a consequence, releases soluble extracellular (ecto-ErbB4) and intracellular (ErbB4-ICD) fragments, by similar proteolytic processing that the described for the Notch receptors (De Strooper et al., 2000), the ApoE/reelin receptor ApoER2 (Balmaceda et al., 2014), and many others. The ectodomain cleavage of ErbB4 by TACE induced in T47-14 cells by 12-O-tetradecanoylphorbol-13acetate (TPA; Vecchi et al., 1996) occurs between His-651 and Ser-652, placing the cleavage site within the ectodomain stalk region approximately 8 residues prior to the transmembrane domain, resulting in a predictable $\sim 80 \mathrm{kDa}$ molecular mass fragment (Cheng et al., 2013). In ErbB4 immunoprecipitates from human CSF we have identified by mass spectrometry peptide fragments from Leu-36 to Lys-606, and by immunoblotting bands of $80 \mathrm{kDa}$, but also of $55 \mathrm{kDa}$. To our knowledge, these shortest ecto-ErbB4 fragments have not been described to date. Interestingly, these shortest ecto-ErbB4 fragments of $\sim 50-55 \mathrm{kDa}$ were the only immunoreactive bands clearly absent in plasma from ErbB4-KO mice, which proves their specificity. Generation of these soluble fragments in cellular model over-expressing the ErbB4-JM-a and the ligand $\mathrm{Nrg} 1$, as well in AAV-Nrg1 SOD1 ${ }^{\mathrm{G} 93 \mathrm{~A}}$ transgenic mice, also implicate increased release of these shortest fragments.

Our determination of ecto-ErbB4 fragments in CSF by western blotting indicated in ALS and in ALS-FTD cases a decrease in the $80 \mathrm{kDa}$ band, the decrease appeared more pronounced for the $55 \mathrm{kDa}$, particularly for the ALS-FTD subgroup. In plasma samples 
from the same subjects, and from the SOD $1^{\mathrm{G} 93 \mathrm{~A}}$ transgenic mice model, the decrease was only significant for the $55 \mathrm{kDa}$ band. However, we should keep in mind that the determination of the $80 \mathrm{kDa}$ band was poorly reliable, at least in plasma, because overlap of unspecific immunoreactivity. Accordingly, we concentrate our analysis in the shortest ectoErbB4 fragments.

The origin and how is generated the shortest ecto-ErbB4 fragment identified in CSF and plasma are presently unknown. In addition to the ErbB4 metalloprotease cleavable and non-cleavable splicing variants JM-a, JM-b, splicing at the cytoplasmic (CYT) locus produces the minor CYT-1 and major CYT-2 variants, respectively, differing only in that 16 amino acids present in CYT-1 are absent in CYT-2 as a result of exon skipping in the latter (Junttila et al., 2000). Thus, alternative splicing do not explain differences in molecular mass for ecto-ErbB4 fragments, and will result only in minor shift in molecular mass of CTF and ICD probably undetectable by electrophoresis/western blotting. Moreover, cleavage of ecto-ErbB4 by TACE or other alternative metalloproteases is expected to occur in similar amino acidic position since similar ErbB4-CTFs have been identified (Lee et al., 2002; Gambarotta et al., 2015). Anyhow, shortest ErbB4-ICD fragment of $\sim 50 \mathrm{kDa}$ has been described in anaplastic large-cell lymphoma cells, highly correlating with MMP9 (Scarfò et al., 2016), a matrix metalloproteinase which can regulate kinase pathways through ErbB receptors (Chattopadhyay and Shubayev, 2009). A 50 kDa ErbB4-ICD, among other smallest C-terminal fragments, have been also identified in human (Chong et al., 2008) and in non-human primate whole brain homogenates and in nuclear extracts, suggesting further or alternative processing for ErbB4 fragments and their functional capacity in the brain (Thompson et al., 2007). Since multiple proteolytic events 
allow for the release of extracellular and cytoplasmic fragments of transmembrane receptors, alternative or further processing of the ecto-ErbB4 cannot be discarded.

Considering that doublets of ecto-ErbB4 fragments are detectable only in plasma and not in CSF, and since the pair of bands are differentially separated during electrophoresis in human and mouse plasma, we have presumed that these differences in electrophoretic migration may be due to differences in glycosylation. Different cell types glycosylate specifically (and differentially) glycoproteins, and glycoforms (and derived fragments) can exhibit differences in apparent molecular weight (Thomson et al., 2016). However, after enzymatic deglycosylation the doublet of shortest ecto-ErbB4 fragments was maintained in human plasma samples. We cannot discard that 50 and $55 \mathrm{kDa}$ are fragments from different glycoforms of ErbB4, since 85 and $80 \mathrm{kDa}$ seem to be the same proteolytic fragment exhibiting differences in glycosylation. We can only speculate that proteolytic events related to generation of shortest ErbB4 fragments, may depend on glycosylation and/or cellular origin. Interestingly, it has been shown that glycosylation can modulate processing of ErbB4 by specific ADAM species (Goth et al., 2015).

Anyhow, in human plasma, in which was possible to discriminate better the doublet in 50 and $55 \mathrm{kDa}$ ecto-ErbB4 fragments, allowing quantification separately, we found that only the $55 \mathrm{kDa}$ was affected during the pathological condition. The $55 \mathrm{kDa}$ band from plasma was the only one, and not the $50 \mathrm{kDa}$, which correlate with the unique shortest ectoErbB4 fragment defined in CSF. Accordingly, we assume that ErbB4 processing (and signaling) is not affect in the same degree in all the tissues during the progression of the ALS. ErbB4 is likely to be the major mediator of Nrg1 function in the brain (Falls, 2003; Wen et al., 2010). Indeed, our data, despite the low number of samples available, indicate that in CSF the largest change occurs in ALS patients suffering FTD. Further studies should 
clarify also if levels of ecto-ErbB4 appeared altered in CSF from subject with FTD with no concomitant occurrence of ALS. The specific tissue preferences of alternative ligand and receptor for ErbB and neuregulins also merit investigation.

Our data, from ALS and ALS-FTD subjects and SOD1 ${ }^{\mathrm{G} 93 \mathrm{~A}}$ transgenic mice, suggest that to determine the levels of proteolytic fragments of ErbB4 can be a suitable read-out of impaired signaling function. Indeed, in brain extracts from SOD ${ }^{\mathrm{G} 93 \mathrm{~A}}$ transgenic mice the level of full-length ErbB4 in cortex was not significantly different from controls, indicating that the decrease in ErbB4-CTF was not a consequence of decreased levels of the proteins. Whether, this decrease of proteolytic processing of ErbB4 depends on decreased levels of specific subtypes of neuregulins or alternative ligands, interference with other proteins or pathological compromised processing, requires clarification. Anyhow, we proposed that quantifying the circulating ecto-ErbB4 fragments maybe useful to estimate an impaired ErbB4 signaling in ALS and ALS+FTD subjects. However, the large overlap for the levels of ecto-ErbB4 fragment between controls and ALS/ALS+FTD prevent their use as a biomarker, at least by determination using western blot. Despite increasing efforts, no biomarkers been identified to facilitate early diagnoses preventing, decelerate, or stop neuronal death in ALS patients. In term to define the potential of the ecto-ErbB4 fragment as a biomarker for ALS and/or ALS-FTD, it will be valuable to replicate our present finding using a more quantitative assay, such as an enzyme linked immunosorbent assay (ELISA). Previous studies have approached the determination by ELISA of the levels of ecto-ErbB4 fragments in medium of cultured cancer cells (Hollmén et al., 2009; 2012). However, prior the application of this desirable outcome it seems necessary to define if an ELISA designed for diagnostic purpose should discriminate between largest and shortest ecto-ErbB4 fragments, and, at least in plasma discriminate between 50 and $55 \mathrm{kDa}$ 
fragments. Anyhow, even the disadvantages of western blotting for quantitative analysis, we consider demonstrated that an impaired ErbB4 signaling is associated with ALS and that the estimation of circulating ecto-ErbB4 fragments can be a reliable approach for an in vivo assessment.

In conclusion, we demonstrated that the existence of circulating $55 \mathrm{kDa}$ ecto-ErbB4 fragments, which decrease in ALS, and particularly in ALS-FTD subjects, may serve to evaluate in vivo an impaired signaling through this receptor. Since ErbB family members are often overexpressed, amplified, or mutated in many forms of cancer (Appert-Collin et al., 2015), determination of circulating of ecto-ErbB4 fragment may also be of applicability as biomarkers for tumor progression and efficiency of therapeutic agents targeting ErbB. 


\section{Footnotes}

We thank Drs. B. Rico and C. Bernard (Centre for Developmental Neurobiology, Institute of Psychiatry, Psychology and Neuroscience, King's College London, London, UK) for generously providing plasma samples from the ErbB4-KO mouse. We also thank (Assumpció). This work was supported by grants from the Fondo de Investigaciones Sanitarias (PI2015/01-4 and PI15/00665 co-funded by the Fondo Europeo de Desarrollo Regional); and through CIBERNED (Instituto de Salud Carlos III, Spain). HZ is a Wallenberg Academy Fellow and is supported by grants from the Swedish and European Research Councils and the UK Dementia Research Institute at UCL. your projects?

Disclosure: All the authors contributed to this work and are in agreement with the findings presented. The authors have no competing interests to disclose in connection with this article. 


\section{References}

Alcolea D, Martínez-Lage P, Sánchez-Juan P, Olazarán J, Antúnez C, Izagirre A, EcayTorres M, Estanga A, Clerigué M, Guisasola MC, Sánchez Ruiz D, Marín Muñoz J, Calero M, Blesa R, Clarimón J, Carmona-Iragui M, Morenas-Rodríguez E, RodríguezRodríguez E, Vázquez Higuera JL, Fortea J, Lleó A. Amyloid precursor protein metabolism and inflammation markers in preclinical Alzheimer disease. Neurology. 2015 Aug 18;85(7):626-33

Alcolea D, Vilaplana E, Suárez-Calvet M, Illán-Gala I, Blesa R, Clarimón J, Lladó A, Sánchez-Valle R, Molinuevo JL, García-Ribas G, Compta Y, Martí MJ, Piñol-Ripoll G, Amer-Ferrer G, Noguera A, García-Martín A, Fortea J, Lleó A. CSF sAPP $\beta$, YKL40, and neurofilament light in frontotemporal lobar degeneration. Neurology. 2017 Jul $11 ; 89(2): 178-188$.

Ancot F, Foveau B, Lefebvre J, Leroy C, Tulasne D. Proteolytic cleavages give receptor tyrosine kinases the gift of ubiquity. Oncogene. 2009 Jun 4;28(22):2185-95.

Appert-Collin A, Hubert P, Crémel G, Bennasroune A. Role of ErbB Receptors in Cancer Cell Migration and Invasion. Front Pharmacol. 2015 Nov 24;6:283.

Balmaceda V, Cuchillo-Ibáñez I, Pujadas L, García-Ayllón MS, Saura CA, Nimpf J, Soriano E, Sáez-Valero J (2014) ApoER2 processing by presenilin-1 modulates reelin expression FASEB J 28:1543-1554.

Bao J, Wolpowitz D, Role LW, Talmage DA. Back signaling by the Nrg-1 intracellular domain. J Cell Biol. 2003 Jun 23;161(6):1133-41. 
Baron AT, Wilken JA, Haggstrom DE, Goodrich ST, Maihle NJ. Clinical implementation of soluble EGFR (sEGFR) as a theragnostic serum biomarker of breast, lung and ovarian cancer. IDrugs. 2009 May;12(5):302-8.

Brooks BR, Miller RG, Swash M, Munsat TL, World Federation of Neurology Research Group on Motor Neuron Diseases. El Escorial revisited: revised criteria for the diagnosis of amyotrophic lateral sclerosis. Amyotroph Lateral Scler Other Motor Neuron Disord. 2000. p. 293-299.

Carpenter G. ErbB-4: mechanism of action and biology. Exp Cell Res. 2003 Mar 10;284(1):66-77.

Chattopadhyay S, Shubayev VI. MMP-9 controls Schwann cell proliferation and phenotypic remodeling via IGF-1 and ErbB receptor-mediated activation of MEK/ERK pathway. Glia. 2009 Sep;57(12):1316-25.

Chen YJ, Zhang M, Yin DM, Wen L, Ting A, Wang P, Lu YS, Zhu XH, Li SJ, Wu CY, Wang XM, Lai C, Xiong WC, Mei L, Gao TM. ErbB4 in parvalbumin-positive interneurons is critical for neuregulin 1 regulation of long-term potentiation. Proc Natl Acad Sci U S A. 2010 Dec 14;107(50):21818-23.

Cheng QC, Tikhomirov O, Zhou W, Carpenter G. Ectodomain cleavage of ErbB-4: characterization of the cleavage site and m80 fragment. J Biol Chem. 2003 Oct $3 ; 278(40): 38421-7$.

Cui B, Cui L, Gao J, Liu M, Li X, Liu C, Ma J, Fang J. Cognitive Impairment in Chinese Patients with Sporadic Amyotrophic Lateral Sclerosis. PLoS One. 2015 Sep 14;10(9):e0137921. 
De Strooper B, Annaert W, Cupers P, Saftig P, Craessaerts K, Mumm JS, Schroeter EH, Schrijvers V, Wolfe MS, Ray WJ, Goate A, Kopan R. A presenilin-1-dependent gamma-secretase-like protease mediates release of Notch intracellular domain. Nature. 1999 Apr 8;398(6727):518-22.

Dols-Icardo O, García-Redondo A, Rojas-García R, Borrego-Hernández D, Illán-Gala I, Muñoz-Blanco JL, Rábano A, Cervera-Carles L, Juárez-Rufián A, Spataro N, De Luna N, Galán L, Cortes-Vicente E, Fortea J, Blesa R, Grau-Rivera O, Lleó A, EstebanPérez J, Gelpi E, Clarimón J. Analysis of known amyotrophic lateral sclerosis and frontotemporal dementia genes reveals a substantial genetic burden in patients manifesting both diseases not carrying the C9orf72 expansion mutation. J Neurol Neurosurg Psychiatry. 2017 Sep 9.

Falls DL. Neuregulins: functions, forms, and signaling strategies. Exp Cell Res. $2003 ; 284: 14-30$.

Gambarotta G, Garzotto D, Destro E, Mautino B, Giampietro C, Cutrupi S, Dati C, Cattaneo E, Fasolo A, Perroteau I. ErbB4 expression in neural progenitor cells (ST14A) is necessary to mediate neuregulin-1beta1-induced migration. J Biol Chem. 2004;279:48808-16.

Gambarotta G, Pascal D, Ronchi G, Morano M, Jager SB, Moimas S, Zentilin L, Giacca M, Perroteau I, Tos P, Geuna S, Raimondo S. Local delivery of the Neuregulin1 receptor ecto-domain (ecto-ErbB4) has a positive effect on regenerated nerve fiber maturation. Gene Ther. 2015 Nov;22(11):901-7. 
Geng F, Zhang J, Wu JL, Zou WJ, Liang ZP, Bi LL, Liu JH, Kong Y, Huang CQ, Li XW, Yang JM, Gao TM. Neuregulin 1-ErbB4 signaling in the bed nucleus of the stria terminalis regulates anxiety-like behavior. Neuroscience. 2016 Aug 4;329:182-92.

Goth CK, Halim A, Khetarpal SA, Rader DJ, Clausen H, Schjoldager KT. A systematic study of modulation of ADAM-mediated ectodomain shedding by site-specific Oglycosylation. Proc Natl Acad Sci U S A. 2015 Nov 24;112(47):14623-8.

Hollmén M, Määttä JA, Bald L, Sliwkowski MX, Elenius K. Suppression of breast cancer cell growth by a monoclonal antibody targeting cleavable ErbB4 isoforms. Oncogene. 2009 Mar 12;28(10):1309-19.

Hollmén M, Liu P, Kurppa K, Wildiers H, Reinvall I, Vandorpe T, Smeets A, Deraedt K, Vahlberg T, Joensuu H, Leahy DJ, Schöffski P, Elenius K. Proteolytic processing of ErbB4 in breast cancer. PLoS One. 2012;7(6):e39413.

Homs J, Pagès G, Ariza L, Casas C, Chillón M, Navarro X, Bosch A. Intrathecal administration of IGF-I by AAVrh10 improves sensory and motor deficits in a mouse model of diabetic neuropathy. Mol Ther Methods Clin Dev. 2014 Jan 15;1:7.

Illan-Gala I, et al. CSF sAPP $\beta$, YKL-40, and NfL along the ALS-FTD spectrum. Under review.

Junttila TT, Sundvall M, Määttä JA, Elenius K. Erbb4 and its isoforms: selective regulation of growth factor responses by naturally occurring receptor variants. Trends Cardiovasc Med. 2000 Oct;10(7):304-10. 
Lasiene J, Komine O, Fujimori-Tonou N, Powers B, Endo F, Watanabe S, Shijie J, Ravits J, Horner P, Misawa H, Yamanaka K. Neuregulin 1 confers neuroprotection in SOD1linked amyotrophic lateral sclerosis mice via restoration of C-boutons of spinal motor neurons. Acta Neuropathol Commun. 2016 Feb 18;4:15.

Lee HJ, Jung KM, Huang YZ, Bennett LB, Lee JS, Mei L, Kim TW. Presenilin-dependent gamma-secretase-like intramembrane cleavage of ErbB4. J Biol Chem. 2002 Feb 22;277(8):6318-23.

Lillo P, Hodges JR. Cognition and behaviour in motor neurone disease. Curr Opin Neurol. 2010 Dec;23(6):638-42.

Loeb JE, Cordier WS, Harris ME, Weitzman MD, Hope TJ. Enhanced expression of transgenes from adeno-associated virus vectors with the woodchuck hepatitis virus posttranscriptional regulatory element: implications for gene therapy. Hum Gene Ther. 1999 Sep 20;10(14):2295-305.

Lynch CC, Vargo-Gogola T, Martin MD, Fingleton B, Crawford HC, Matrisian LM. Matrix metalloproteinase 7 mediates mammary epithelial cell tumorigenesis through the ErbB4 receptor. Cancer Res. 2007 Jul 15;67(14):6760-7.

Ma Z, Li Q, An H, Pankonin MS, Wang J, Loeb JA. Targeting human epidermal growth factor receptor signaling with the neuregulin's heparin-binding domain. J Biol Chem. 2009 Nov 13;284(46):32108-15.

Määttä JA, Sundvall M, Junttila TT, Peri L, Laine VJ, Isola J, Egeblad M, Elenius K. Proteolytic cleavage and phosphorylation of a tumor-associated ErbB4 isoform 
promote ligand-independent survival and cancer cell growth. Mol Biol Cell. 2006 Jan;17(1):67-79.

Mancuso R, Oliván S, Osta R, Navarro X. Evolution of gait abnormalities in SOD1(G93A) transgenic mice. Brain Res. 2011 Aug 11;1406:65-73

Mancuso R, Martínez-Muriana A, Leiva T, Gregorio D, Ariza L, Morell M, Esteban-Pérez J, García-Redondo A, Calvo AC, Atencia-Cibreiro G, Corfas G, Osta R, Bosch A, Navarro X. Neuregulin-1 promotes functional improvement by enhancing collateral sprouting in SOD1(G93A) ALS mice and after partial muscle denervation. Neurobiol Dis. 2016 Nov;95:168-78.

Montuschi A, Iazzolino B, Calvo A, Moglia C, Lopiano L, Restagno G, Brunetti M, Ossola I, Lo Presti A, Cammarosano S, Canosa A, Chiò A. Cognitive correlates in amyotrophic lateral sclerosis: a population-based study in Italy. J Neurol Neurosurg Psychiatry. 2015 Feb;86(2):168-73.

Ni CY, Murphy MP, Golde TE, Carpenter G. gamma -Secretase cleavage and nuclear localization of ErbB-4 receptor tyrosine kinase. Science. 2001 Dec 7;294(5549):21798.

Pascal D, Giovannelli A, Gnavi S, Hoyng SA, de Winter F, Morano M, Fregnan F, Dell'Albani P, Zaccheo D, Perroteau I, Pellitteri R, Gambarotta G. Characterization of glial cell models and in vitro manipulation of the neuregulin1/ErbB system. Biomed Res Int. 2014;2014:310215. 
Piedra J, Ontiveros M, Miravet S, Penalva C, Monfar M, Chillon M. Development of a rapid, robust, and universal picogreen-based method to titer adeno-associated vectors. Hum Gene Ther Methods. 2015 Feb;26(1):35-42.

Plowman GD, Culouscou JM, Whitney GS, Green JM, Carlton GW, Foy L, Neubauer MG, Shoyab M. Ligand-specific activation of HER4/p180erbB4, a fourth member of the epidermal growth factor receptor family. Proc Natl Acad Sci U S A. 1993 Mar 1;90(5):1746-50.

Portelius E, Brinkmalm G, Tran A, Andreasson U, Zetterberg H, Westman-Brinkmalm A, Blennow K, Ohrfelt A. Identification of novel N-terminal fragments of amyloid precursor protein in cerebrospinal fluid. Exp Neurol. 2010 Jun;223(2):351-8.

Roskoski R Jr. The ErbB/HER family of protein-tyrosine kinases and cancer. Pharmacol Res. 2014 Jan;79:34-74.

Rio C, Buxbaum JD, Peschon JJ, Corfas G. Tumor necrosis factor-alpha-converting enzyme is required for cleavage of erbB4/HER4. J Biol Chem. 2000 Apr $7 ; 275(14): 10379-87$.

Ripps ME, Huntley GW, Hof PR, Morrison JH, Gordon JW. Transgenic mice expressing an altered murine superoxide dismutase gene provide an animal model of amyotrophic lateral sclerosis. Proc Natl Acad Sci U S A. 1995 Jan 31;92(3):689-93.

Sanderson MP, Dempsey PJ, Dunbar AJ. Control of ErbB signaling through metalloprotease mediated ectodomain shedding of EGF-like factors. Growth Factors. 2006 Jun;24(2):121-36. 
Sardi SP, Murtie J, Koirala S, Patten BA, Corfas G. Presenilin-dependent ErbB4 nuclear signaling regulates the timing of astrogenesis in the developing brain. Cell. 2006 Oct 6;127(1):185-97.

Scarfò I, Pellegrino E, Mereu E, Kwee I, Agnelli L, Bergaggio E, Garaffo G, Vitale N, Caputo M, Machiorlatti R, Circosta P, Abate F, Barreca A, Novero D, Mathew S, Rinaldi A, Tiacci E, Serra S, Deaglio S, Neri A, Falini B, Rabadan R, Bertoni F, Inghirami G, Piva R; European T-Cell Lymphoma Study Group. Identification of a new subclass of ALK-negative ALCL expressing aberrant levels of ERBB4 transcripts. Blood. 2016 Jan 14;127(2):221-32.

Strong MJ, Abrahams S, Goldstein LH, et al. Amyotrophic lateral sclerosis frontotemporal spectrum disorder (ALS-FTSD): Revised diagnostic criteria. Amyotroph Lateral Scler Frontotemporal Degener. 2017;18:153-174.

Takahashi Y, Fukuda Y, Yoshimura J, Toyoda A, Kurppa K, Moritoyo H, Belzil VV, Dion PA, Higasa K, Doi K, Ishiura H, Mitsui J, Date H, Ahsan B, Matsukawa T, Ichikawa Y, Moritoyo T, Ikoma M, Hashimoto T, Kimura F, Murayama S, Onodera O, Nishizawa M, Yoshida M, Atsuta N, Sobue G; JaCALS, Fifita JA, Williams KL, Blair IP, Nicholson GA, Gonzalez-Perez P, Brown RH Jr, Nomoto M, Elenius K, Rouleau GA, Fujiyama A, Morishita S, Goto J, Tsuji S. ERBB4 mutations that disrupt the neuregulin-ErbB4 pathway cause amyotrophic lateral sclerosis type 19. Am J Hum Genet. 2013 Nov 7;93(5):900-5. 
Thompson M, Lauderdale S, Webster MJ, Chong VZ, McClintock B, Saunders R, Weickert CS. Widespread expression of ErbB2, ErbB3 and ErbB4 in non-human primate brain. Brain Res. 2007 Mar 30;1139:95-109.

Tidcombe H, Jackson-Fisher A, Mathers K, Stern DF, Gassmann M, Golding JP. Neural and mammary gland defects in ErbB4 knockout mice genetically rescued from embryonic lethality. Proc Natl Acad Sci U S A. 2003 Jul 8;100(14):8281-6.

Thomson RB, Thomson CL, Aronson PS. N-glycosylation critically regulates function of oxalate transporter SLC26A6. Am J Physiol Cell Physiol. 2016 Dec 1;311(6):C866C873.

Vecchi M, Carpenter G. Constitutive proteolysis of the ErbB-4 receptor tyrosine kinase by a unique, sequential mechanism. J Cell Biol. 1997 Nov 17;139(4):995-1003.

Vecchi M, Baulida J, Carpenter G. Selective cleavage of the heregulin receptor ErbB-4 by protein kinase C activation. J Biol Chem. 1996 Aug 2;271(31):18989-95.

Wen L, Lu YS, Zhu XH, Li XM, Woo RS, Chen YJ, Yin DM, Lai C, Terry AV Jr, Vazdarjanova A, Xiong WC, Mei L. Neuregulin 1 regulates pyramidal neuron activity via ErbB4 in parvalbumin-positive interneurons. Proc Natl Acad Sci U S A. 2010 Jan $19 ; 107(3): 1211-6$.

Woo RS, Li XM, Tao Y, Carpenter-Hyland E, Huang YZ, Weber J, Neiswender H, Dong XP, Wu J, Gassmann M, Lai C, Xiong WC, Gao TM, Mei L. Neuregulin-1 enhances depolarization-induced GABA release. Neuron. 2007 May 24;54(4):599-610. 
Xiao X, Li J, Samulski RJ. Production of high-titer recombinant adeno-associated virus vectors in the absence of helper adenovirus. J Virol. 1998 Mar;72(3):2224-32.

Yu WH, Woessner JF Jr, McNeish JD, Stamenkovic I. CD44 anchors the assembly of matrilysin/MMP-7 with heparin-binding epidermal growth factor precursor and ErbB4 and regulates female reproductive organ remodeling. Genes Dev. 2002 Feb 1;16(3):307-23.

Zeng F, Zhang MZ, Singh AB, Zent R, Harris RC. ErbB4 isoforms selectively regulate growth factor induced Madin-Darby canine kidney cell tubulogenesis. Mol Biol Cell. 2007 Nov;18(11):4446-56.

Zolotukhin S, Byrne BJ, Mason E, Zolotukhin I, Potter M, Chesnut K, Summerford C, Samulski RJ, Muzyczka N. Recombinant adeno-associated virus purification using novel methods improves infectious titer and yield. Gene Ther. 1999 Jun;6(6):973-85. 


\section{Figure Legends}

Figure 1. Ecto-ErbB4 fragments are present in CSF and plasma. (A) Schematic representation of ErbB4 including domain organization (adapted from Zeng et al., 2007; Hollmén et al., 2012). ErbB4 glycoproteins is a type I transmembrane receptor tyrosine kinase with an extracellular region containing two extracellular cysteine-rich (CR1 and CR2) domains, a transmembrane domain (TM) flanked by short intracellular juxtamembrane (JM) regions, the extracellular contains alternative JM isoforms, with (JMa) or without (JM-b) a proteolytic TACE cleavage site between His651 and Ser652. The approximate cleavage site by $\gamma$-secretase is also indicate. The intracellular region including a tyrosine kinase domain, a regulatory domain with autophosphorylation sites plus sequence changes attributable to the CYT-1 and CYT-2 isoforms (CYT-2 isoform has a 16amino acid deletion that contains a PI3K-binding and a WW domain binding motif) and the carboxyl-terminal tail. The approximate localizations matching identified CSF-ADAM10 peptides by mass spectrometry is indicated (Hansson et al., 2017). (B) Western blot of human CSF samples from control subjects. Control CSF samples were also immunoprecipitated and precipitated (IP) and unbound proteins (Unb) were immunoblotted with the indicated anti-ErbB4 antibodies, or resolved by mass spectrometry. Eighteen tryptic peptides spanning aa 36-606, i.e., most of the N-terminal half of ErbB4 (Uniprot entry Q15303, ERBB4_HUMAN), were detected with high confidence (see above for approximate localizations and supplemental Table 1 for details). Extracts incubated with a rabbit IgG (IPc) were analyzed in parallel as negative controls. Arrowhead indicates a nonspecific band. (C) Control human plasma samples were also resolved by western blotting 
and immunoprecipitated as indicated. (D) Additionally, human samples were resolved by SDS-PAGE prior and after enzymatic deglycosylation (deGlyc).

\section{Figure 2. Shortest ecto-ErbB4 fragments are specific and generated in presence of}

Nrg1. (A) Plasma samples from wild-type mice were blotted with a polyclonal anti-Nterminal ErbB4 antibody. ErbB4-KO mice (KO) and control littermates (Ctrl) were also blotted with the same ErbB4 antibody. In general, immunoreactivities of the bands corresponding to largest ecto-ErbB4 complexes were weak in ErbB4-KO mouse than in control littermates, but the shortest bands were the unique absent. (B) HEK cells were transfected with the full-length cleavable ErbB4 JM-a (ErbB4), and some also with the human Nrg1-III (Nrg1). ErbB4 in the cell extracts and soluble ErbB4 in the medium were assayed in western blots using anti C-terminal or $\mathrm{N}$-terminal polyclonal antibodies respectively (equivalent amounts of protein of the cell extracts and equal volumes of medium were loaded in each lane). GAPDH served as a loading control for cellular extracts. The N-terminal antibody detects the largest (NTF-large) and shortest (NTF-short) soluble N-terminal fragments in cellular medium. The C-terminal antibody served to confirm ErbB4 over-expression in transfected cells (ErbB4 full-length: ErbB4-fk). Representative blots are shown.

Figure 3. Decreased ecto-ErbB4 fragments in ALS CSF. (A) Representative blot, probed with the polyclonal anti-N-terminal ErbB4 antibody, and (B) densitometric quantification of the immunoreactivity from the $\sim 80$ and $55 \mathrm{kDa}$ ecto-ErbB4 fragments in CSF samples from 20 ALS (open circle; including 5 ALS with concomitant FTD: ALS-FTD, open 
triangle) and 10 age-matched control subjects (Ctrl; closed circle). Arrowhead indicates a non-specific band. The data represent the means \pm SEM; $p$ values are shown.

Figure 4. Decreased ecto-ErbB4 fragments in ALS plasma. (A) Representative blot, probed with anti-N-terminal ErbB4 antibody, and (B) densitometric quantification of the immunoreactivity from the $\sim 85,80,55$ and $50 \mathrm{kDa}$ ecto-ErbB4 fragments in plasma samples from the same individuals than in Fig. 3, 19 ALS (open circle; including 5 ALS with concomitant FTD: ALS-FTD, open triangle) and 10 age-matched control subjects (Ctrl; closed circle). Arrowhead indicates a non-specific band. The data represent the means \pm SEM; $p$ values are shown (n.s.: non significant).

Figure 5. Decreased levels of $55 \mathrm{kDa}$ ecto-ErbB4 fragments in plasma from the SOD1 ${ }^{\text {G93A }}$ transgenic mice. (A) Representative blot and (B) densitometric quantification of the $55 \mathrm{kDa}$ ecto-ErbB4 band in plasma samples from 12 month-old transgenic (SOD1$\mathrm{Tg} ; \mathrm{n}=10)$ and wild-type littermates $(\mathrm{Ctrl} ; \mathrm{n}=10)$ animals. Arrowhead indicates a nonspecific band. The data represent the means \pm SEM; $p$ value is shown. (C) Eight month-old SOD1-Tg $(n=8)$ were injected with an AAV encoding for soluble ectodomain of Nrg1-III and analyzed the plasma levels of Nrg1-III (ecto-Nrg1) and ecto-ErbB4 fragments after sacrifice (16-month of age); (D) showing a positive correlation. Linear regression coefficient $(\mathrm{R})$ and $p$ value are shown.

Figure 6. Decreased generation of C-terminal ErbB4 fragments in brain from the SOD1 ${ }^{\text {G93A }}$ transgenic mice. (A) $\mathrm{CHO}$ cell were transfected with ErbB4 (+) or with the pcDNA3 expression plasmid as a control (-) and resolved with a C-terminal antibody for 
ErbB4 in order to resolve the reliable C-terminal fragment of ErbB4 (ErbB4-CTF). Western blots of brain extracts from 12 month-old transgenic (SOD1-Tg; $\mathrm{n}=5$ ) and wild-type littermates $(\mathrm{Ctrl} ; \mathrm{n}=5)$ animals, were also probed for ErbB4 C-terminal and GAPDH immunoreactivities. (B) Densitometric quantification of the full-length ErbB4 (ErbB4-fl; $\sim 160 \mathrm{kDa}$ ) were normalized to GAPDH staining intensity. (C) The quotient derived from the immunoreactivity for the ErbB4-CTF ( 80 kDa) relative to that for the ErbB4-fl in each sample [ErbB4-CTF/ErbB4-fl] reflect altered proteolytic processing of ErbB4 in SOD1-Tg. The data represent the means $\pm \mathrm{SEM} ; p$ values are shown.

Supplemental Figure 1. Positive correlation between immunoreactivity levels of the ecto-ErbB4 fragments in CSF and samples. (A) Lineal correlation between ecto-ErbB4 fragments from CSF and (B) plasma samples from 10 age-matched controls (Ctrl: closed circle) and 20 ALS patients (19 for plasma samples, represented with open circle; 5 ALS+FTD open triangle) were assayed for N-terminal ErbB4 (see Fig. 3 and 4). (C) Correlation between the $55 \mathrm{kDa}$ ecto-ErbB4 fragment from CSF and plasma is also shown. Correlations between indicated ecto-ErbB4 fragments in Ctrl subjects are represented as solid lines and for ALS patients as dotted lines. Linear regression coefficient (R) and $p$ values for each correlation are shown (n.s.: non significant). 
Supplemental Table 1. Identified peptides from ErbB4 in human CSF.

\begin{tabular}{|c|c|c|c|c|c|c|}
\hline Positions & Sequence & Modifications ${ }^{a}$ & $\begin{array}{l}\text { \# Missed } \\
\text { Cleavages }\end{array}$ & $\begin{array}{c}{[\mathrm{M}+\mathrm{H}]^{+}} \\
{[\mathrm{Da}]}\end{array}$ & $\begin{array}{l}\text { Mascot } \\
\text { Score }\end{array}$ & $\begin{array}{l}\text { Expectation } \\
\text { Value }^{\mathrm{b}}\end{array}$ \\
\hline $036-047$ & LSSLSDLEQQYR & & 0 & 1438.72 & 107 & 4.53E-10 \\
\hline 082-095 & EVTGYVLVALNQFR & & 0 & 1608.87 & 78 & 2.17E-07 \\
\hline 096-103 & YLPLENLR & & 0 & 1017.57 & 47 & $6.27 \mathrm{E}-04$ \\
\hline $115-124$ & YALAIFLNYR & & 0 & 1243.68 & 49 & 3.37E-04 \\
\hline $126-137$ & DGNFGLQELGLK & & 0 & 1290.67 & 54 & $1.30 \mathrm{E}-04$ \\
\hline $154-168$ & FLCYADTIHWQDIVR & 1xCarbamidomethyl [C3] & 0 & 1936.94 & 60 & $1.85 \mathrm{E}-05$ \\
\hline $197-210$ & CWGPTENHCQTLTR & 2xCarbamidomethyl [C1; C9] & 0 & 1759.76 & 92 & 4.97E-09 \\
\hline $221-232$ & CYGPYVSDCCHR & 3xCarbamidomethyl [C1; C9; C10] & 0 & 1573.60 & 102 & 8.59E-11 \\
\hline $283-291$ & YTYGAFCVK & 1xCarbamidomethyl [C7] & 0 & 1108.51 & 37 & $4.13 \mathrm{E}-03$ \\
\hline $292-306$ & KCPHNFVVDSSSCVR & 2xCarbamidomethyl [C2; C13] & 1 & 1791.83 & 66 & $4.83 \mathrm{E}-06$ \\
\hline 293-306 & CPHNFVVDSSSCVR & 2xCarbamidomethyl [C1; C12] & 0 & 1663.73 & 84 & $3.60 \mathrm{E}-08$ \\
\hline $322-332$ & MCKPCTDICPK & $\begin{array}{c}\text { 1xOxidation [M1]; } \\
\text { 3xCarbamidomethyl [C2; C5; C9] }\end{array}$ & 0 & 1425.60 & 58 & $1.01 \mathrm{E}-05$ \\
\hline $322-332$ & MCKPCTDICPK & 3xCarbamidomethyl [C2; C5; C9] & 0 & 1409.60 & 56 & $1.98 \mathrm{E}-05$ \\
\hline $333-355$ & ACDGIGTGSLMSAQTVDSSNIDK & $\begin{array}{c}\text { 1xOxidation [M11]; } \\
\text { 1xCarbamidomethyl [C2] }\end{array}$ & 0 & 2343.04 & 29 & $1.05 \mathrm{E}-02$ \\
\hline $439-451$ & QQGITSLQFQSLK & & 0 & 1477.80 & 51 & $1.61 \mathrm{E}-04$ \\
\hline $531-544$ & ICIESCNLYDGEFR & 2xCarbamidomethyl [C2; C6] & 0 & 1775.77 & 90 & 5.69E-09 \\
\hline $580-592$ & CSHFKDGPNCVEK & 2xCarbamidomethyl [C1; C10] & 1 & 1577.68 & 58 & 7.97E-06 \\
\hline $585-606$ & DGPNCVEKCPDGLQGANSFIFK & 2xCarbamidomethyl [C5; C9] & 1 & 2453.12 & 109 & $1.23 \mathrm{E}-10$ \\
\hline $593-606$ & CPDGLQGANSFIFK & 1xCarbamidomethyl [C1] & 0 & 1553.74 & 91 & $1.63 \mathrm{E}-08$ \\
\hline
\end{tabular}

${ }^{\text {a }}$ Carbamidomethyl modifications at Cys residues are due to the blocking using IAA prior to digestion with trypsin.

${ }^{b}$ The expectation value is the probability that the peptide identification is a random positive false. 